\title{
IMPLEMENTATION OF PRIVACY SECURED SMART METER FOR DEMAND SIDE MANAGEMENT
}

\author{
K.R.Sindhuja ${ }^{1}$, N.Sampathraja ${ }^{2}$ \\ ${ }^{1} P G$ Scholar, Department of Electrical and Electronics Engineering, PSG College of Technology, Coimbatore, India \\ ${ }^{2}$ Assistant Professor, Department of Electrical and Electronics Engineering, PSG College of Technology, Coimbatore, \\ India
}

\begin{abstract}
To develop a prototype model of smart meter for the purpose of demand-side management. This work exchange sensitive data transfer between energy producer \& the consumer, Thereby aims at increasing the efficiency of power consumption. One of the intent of DSM is to educate the consumer to take part in improving the peak load management by focusing the time of usage of load consumption. This smart meter transfer data to control centre wirelessly through IoT modem. . IoT V2 SIM-900 which sends data at a sampling rate of 56-114 kbps by a packet based wireless communication to the webpage through internet. The webpage which is created by an open source software www.iot cloud servers.com. The IoT modem not only acquiring data from the sensor to the cloud but also monitor such information and alert the users whenever the data sending route got damaged. In this work, the smart meters are communicated and monitored by one another and by the control center via UART (Universal Asynchronous Receiver Transmitter) to detect compromise attack, which uses Arduino UNO as a controller. Usually software can't protect hardware from physical attack. A little bit of Physical access to the smart meter is so dangerous, causes pwning, mess up with the memory and finally leads to faulty data reading. Buses are used in an embedded system to communicate with each other. Most commonly used protocols are I2C, UART and SPI. Whenever the bus protocol of a particular meter gets attacked/damaged. It couldn't send data to the main server. At that time, the data's are bypassed and safely send via neighbouring meter to the control centers. During this process is taking place the neighbouring meter couldn't see defected meters data, they can only do data transfer functions.
\end{abstract}

Keywords: - IoT modem V2 SIM900, Arduino UNO, By-passing protocol, Web page creating.

\section{INTRODUCTION}

As the development in Internet of Things which led all the embedded devices to be enabled with web services which is simply can also be termed as "Things Speak". This IoT improvement will help various societal challenges like connected cars will help reduces traffic, pollution and Connecting trees will helps to reduce deforestation etc. Like this connected devices will finally reduce the carbon footprint [13]. Implementing IoT enabled smart meter will takes the technology to the next level. The internet of things was first evolved in Auto Id method. Earlier if all the daily objects in the world will be equipped with barcode computer will able to easily identify it in this way IoT got developed. The IoT which is being already implemented in various locality having mobility with large number of devices connected wirelessly and are miniature in size with the great capacity of interoperability [11].

\subsection{History of Smart Meter}

In the year 1972, electro-mechanical meters was first developed to measure electricity with the help of magnetic disk and it's been deployed for so many years as they can provide accurate readings its major drawback is that they cannot measure the load with the time of day consumption [1]. Thus the traditional meters are replaced with solid state devices which has several advantages like they can measure reactive power and power factor but it also have some disadvantages like they cannot measure data and store it.

The regular meters of gas and electricity can be measured only through the quantity of energy utilized and further the details regarding the period of consumption. But the smart meters could be able to provide inherent information's relating to the consumers energy along with the real time measurement details of electrical consumption to the consumers as well as the power suppliers and can also be stored [10]. Thus the Smart meters in-depth can read the current flow in any electrical circuits and thereby identify the problems that would arise on the account of its fluctuation. Smart meters, literally denote devices intended for measurement of electricity which are normally in usage to provide data in papers to the consumers and also carry out total maintenance of the system.

\subsection{Motivation}

One of the greatest possible milestone is that India is having 200 million smart meters at present and they are planning to install further 130 million smart meter within the year 2021 [3]. To top it off the government is planning to invest 21 billion dollar until 2025 in smart grid technologies. Along 
with that 14 smart cities pilot projects has been launched in India to explore smart solution in generation and distribution sector.

As might be expected, Indian market is heading towards long term opportunities and solution in smart metering and its concepts. The aim is to restrain the electricity theft, reduce distribution and transmission losses to acceptable standards and have better management of supply based on analysis of real time demand. It is also estimated that within the year 2020 every house in UK will have individual smart meters

\subsection{Problem Formulation}

By the advancement and automation of smart meter there are numerous problems which also need to be addressed. As there is already a power line to smart meter, additional wire to communication will become more congested. So considering the meter go wireless in nature and able to work in a mesh network capable of reporting data from the most far away meters. Hence it is self-forming to give best results. In India, meters are usually are just one way communication. This causes the service providers not able to identify losses and real time power consumption. In wireless communication, the gateway plays a vital role in it. The highest communication medium has high penetrating power. Among various communication medium internet protocol has high penetrating power [6]. In case of any fault, technicians are needed to identify what actual damage has taken part. But with the facility of interoperability, meter can communicate with each other [7]. Most of the times billing can be divided into peak hours and normal hours. Without the proper education customers will tend to consume more no of units at peak hours. As they cannot visualize their pattern and they can't manage the power finally billing will be much expensive.

\subsection{Objective}

- To design and develop a smart meter that transfer current and voltage data's wirelessly.

- Bypassing the signal to control unit through nearby smart meter at the time of any fault.

- To minimize the energy consumption by graphically display the load pattern.

\section{PROPOSED METHOD}

Smart meter vary from each other in some aspects like two way communication, regular data reading rate etc. The basic function of a smart meter is to record current, voltage, max demand, real power, reactive power. The proposed smart meter are characterized by the following

- Two way communication between the customer and the utility through IoT modem

- Measure electrical parameters and display it in LCD

- Record load data in the Web page
- Bypassing the customers load data through another meter whenever any damages taking place.

- In addition in built storage capability

Further on, by the evaluation of the technologies nowadays smart meter are being manufacture with additional features. Certain in-depth additional features in this meter are as follows

- Demand Control capability

- Programmable sampling rate

- Automatic fault detection and user indication

The various sub-blocks blocks of the proposed model includes Current and voltage sensing unit, Data acquisition, processing and controlling unit, Power supply unit, Wireless transferring and real time clock unit, Communication module

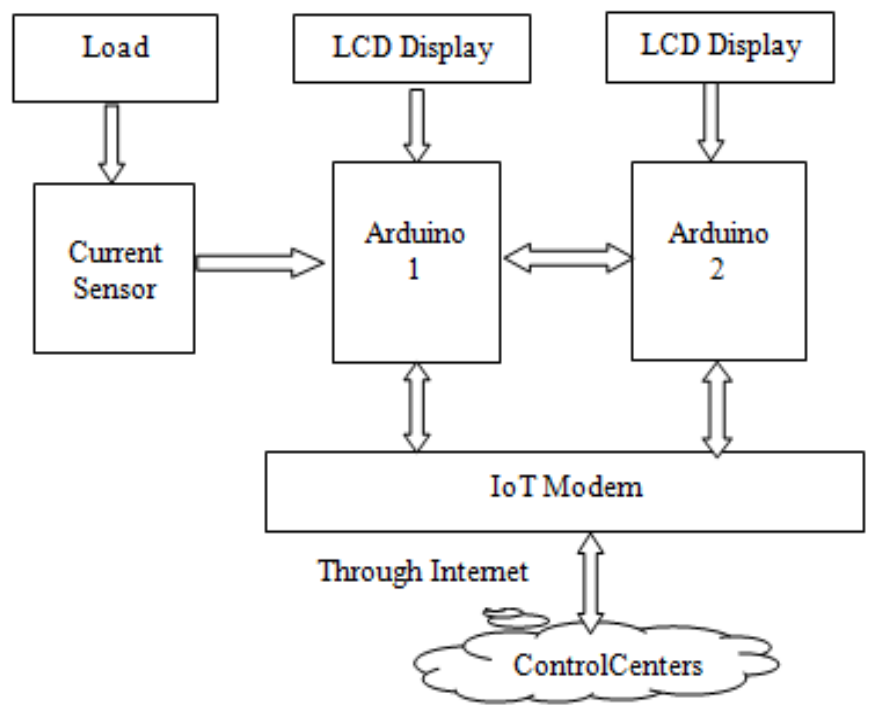

Fig 1: Block Diagram of the Proposed Model

\subsection{Power Supply Unit}

The power supply to the experimental setup is giver based on the Fig.2 One of the main part of this work is the power supply. Thus the single phase supply of $230 \mathrm{~V} / 120 \mathrm{~V}$ is given to step down transformer which lowers the voltage and its secondary voltage will be $12 \mathrm{~V}$ (230:12 V ratio).

The $12 \mathrm{~V}$ ac supply is converted into $12 \mathrm{~V}$ direct current with the help of diode bridge rectifier. Hence a separate 12 $\mathrm{V}$ unit required to IoT modem. The $12 \mathrm{~V}$ dc which is given to IoT modem version 2 SIM 900 as it has inbuilt $12 \mathrm{~V}$ to 5 $\mathrm{V}$ regulator. The $12 \mathrm{~V}$ dc supply is separately given to the regulator that converts fluctuating $12 \mathrm{~V}$ dc to straight $5 \mathrm{~V}$ dc without any flickers. The main supply from $5 \mathrm{~V}$ is given to all devices like two arduino, two LCD display and the current sensing unit. As it has power line and communication line to reduce its complication. The power line from $5 \mathrm{~V} \mathrm{dc}$ is pasted in a dot board and the overall components are pasted in a wooden cardboard. 
Thus the $5 \mathrm{~V}$ dc supply is given to all the device. Further it also have some communication wirings like the two smart meters i.e. the arduino will have communication interaction with the help UART (universal asynchronous receiver transmitter) cable.

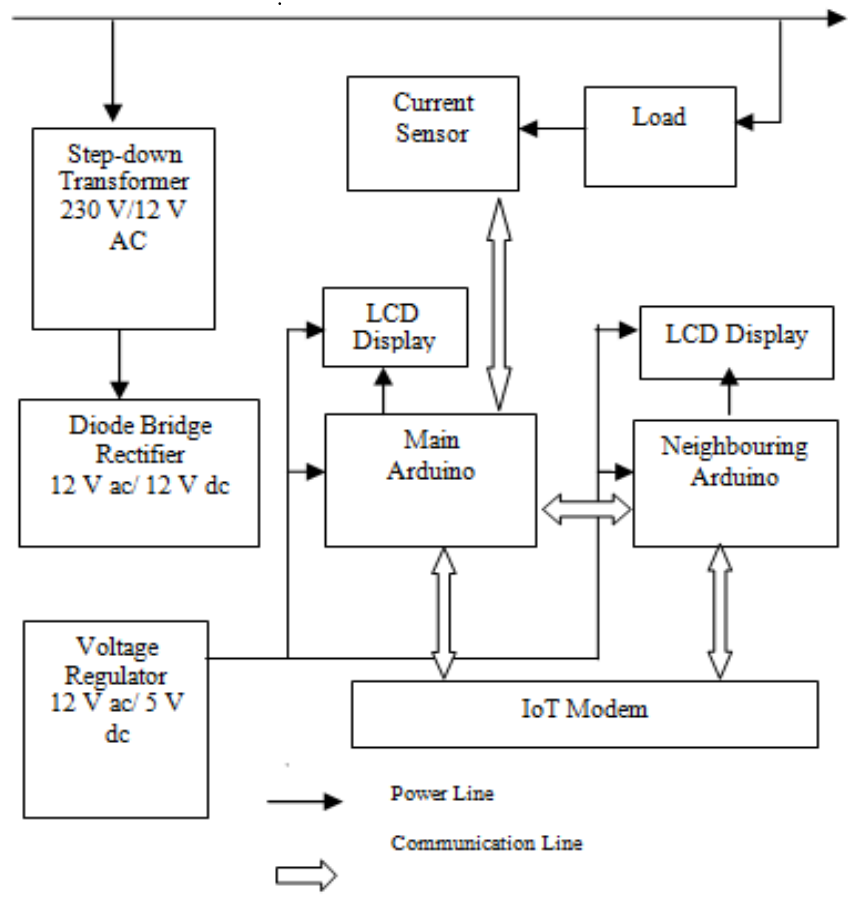

Fig 2: Power Supply of the Hardware Unit

The usage of load of $100 \mathrm{~W}$ lamp is sensed, measured and transferred to the smart meter of arduino through the current sensing unit. The two arduino which also communicates to IoT modem through this communication cable beyond this the data transfer will be done through wireless medium.

\subsection{Data Accquistion, Processing and Control Unit}

The meter to meter connection and its IoT modem wiring connection is given based on the fig. 3 .

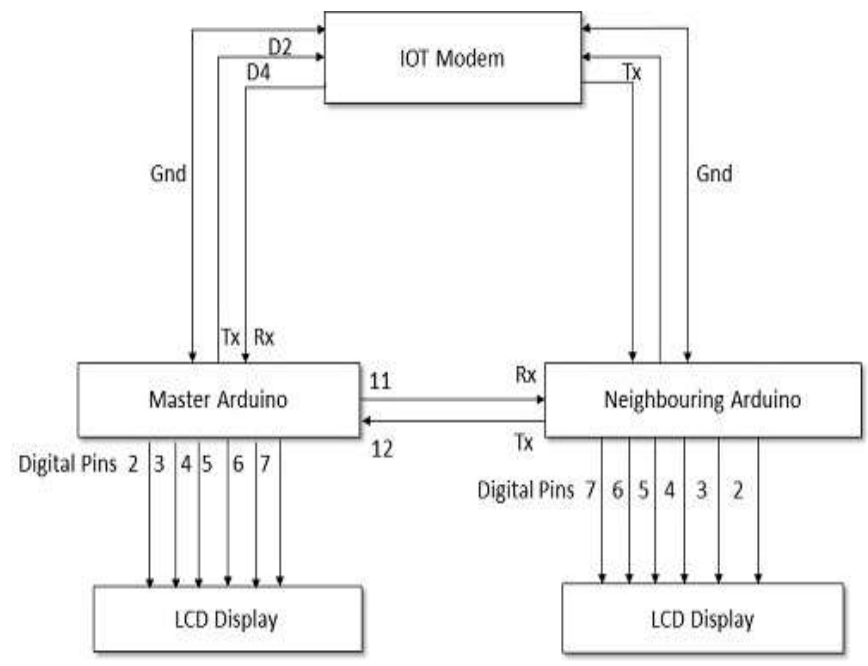

Fig 3: Meter to Meter Communication
The two LCD's are connected to the respective Arduino's with the help of pot. The aim of this work is to By-pass the data flow at the time of master meter of arduino which incur any damage in data transferring. Each arduino transfer data at a specified baud rate. The standard baud rate of 9600 is used. Baud rate is nothing data sending speed over a serial line it can be measured in bits per second.

At first data's from the load sensor will send to the main arduino at a rate 9600 baud rate which can be displayed in LCD. This data are transferred to the IoT modem for the purpose of wireless transfer. When the data's are transferred to IoT modem from main arduino there won't be any problem. Whenever at a time of any fault and arduino couldn't send data at that time, the main arduino pins of 11 and 12 will be enabled which set as a transmitting and receiving pins and they are connected to $\mathrm{Tx}$ and $\mathrm{Rx}$ pin of the arduino this by-pass path will be enabled at the time of any fault. Finally at the time of any fault the meter data's will be bypassed and send over a neighbouring energy while this entire process is taking place the neighbouring meter will not be able to see the faulty meters data.

\subsection{IoT Modem Configuration}

The IoT SIM 900 modem connected Atmega328p controller has its own protocol. The Arduino UNO board and the modem is connected with a UART cable. A Software serial library pin which enables GPIO pin as Tx and Rx for serial communication and other arduino will transfer through its Tx and Rx pin itself. After establishing the connection between the modem and the two Arduino's, the modem and its web server is to be connected. For connecting the GPRS to the internet, Access Point Name - APN is needed .It is considered as a gateway for any devices to connect to the Internet [12]. Thus the airtel sim card is used as a gateway, the APN for airtel network is "airtelgprs.com".

Once the gateway connection with the modem is created it will receive an IP address on the internet.

After receiving the IP address. Specify the user name and password, based upon getting the correct user name and password the modem will initiate a connection with the web server.

As the device's handshake is done. The data's of the sensor will streaming onto the webserver through modem at a time interval of 10 to 12 seconds per iteration and its frequencies is limited based upon GPRS connection capability [8].

Command for initializing GSM

AT: This command is used to check whether GSM module is responding or not.

AT+CPIN: this command is used to check whether SIM card is inserted in GSM Module or not[13]. 
AT+CIPSHUT - to close TCP Port Explicitly means disconnecting connection

AT+CGATT - Checking SIM card has internet connection or not

$\mathrm{AT}+\mathrm{CSTT}=$ "APN", "username", "Password" - connect to internet (ex: AT+CSTT="airtelgprs.com”)

AT+CIICR - bring up the wireless network. Checking SIM card has data pack or balance.

AT+CIFSR - get IP (sometimes without this command GSM do not work)

AT+CIPSTART ="TCP",'SERVER IP",,PORT". It is used for creating TCP connection with the server that is provide in place of SERVER IP

AT+CIPSEND - this command is used for sending data to the server.

\subsection{Development of Web Page}

The web page is created with the help of open source software www.iot cloud servers. Com. In that webpage the dashboards were created. In that dashboard the current, voltage, power and power factor values are supposed to display both real time values and also displayed graphically. There also available facilities like sending mail and sms to the customers.

After creating the Webpage, the IoT modem and the website can be linked with the help of Terminal.exe software. With the help of TTL cable (i.e. which connect the IoT modem wire to the laptop through usb cable) already created user name and password is fed and network connection is established[7].

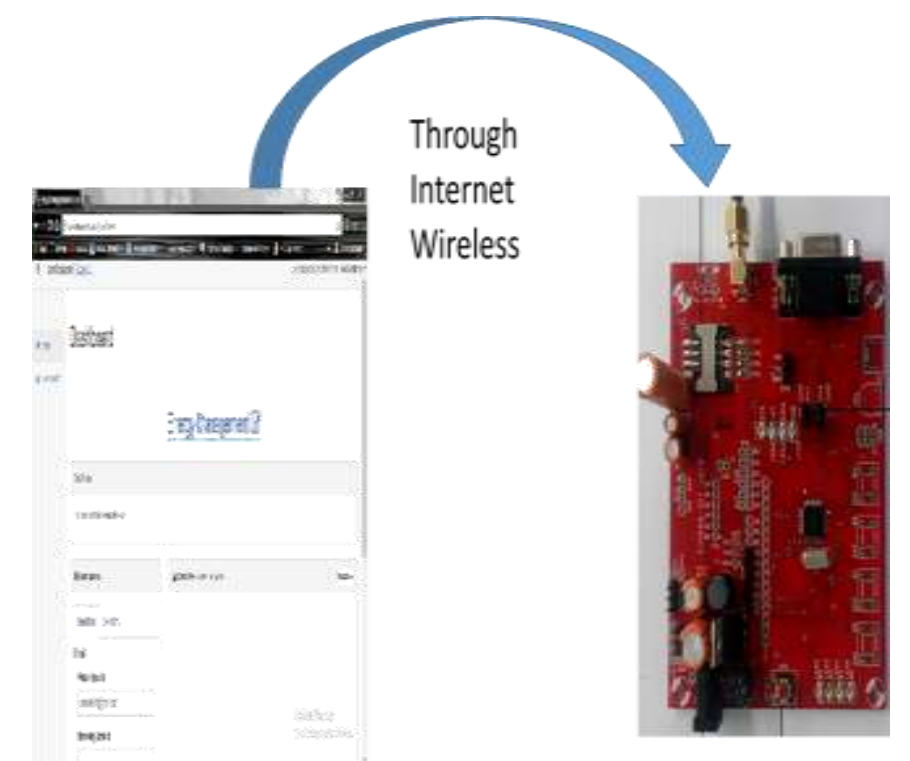

Fig 4: Web Page Development

\section{SIMULATION RESULT}

Simulation was done using a proteus software. As this experiment uses an arduino UNO with the controller of Atmega832. For current sensing ACS712 sensor is used in simulation. But in hardware ZMCT103 wound coil type sensor is used as it gives better results in variation of load. The components needed for simulation results are LM044L LCD Display, Arduino UNO, Current Sensor, Power Supply, Lamp Load.

It can be seen that the $100 \mathrm{~W}$ lamp load is powered with the help of battery. This load is sensed with the help of current sensor and is send to arduino after proper calibration and Analog to digital conversion is been displayed in the LCD display.

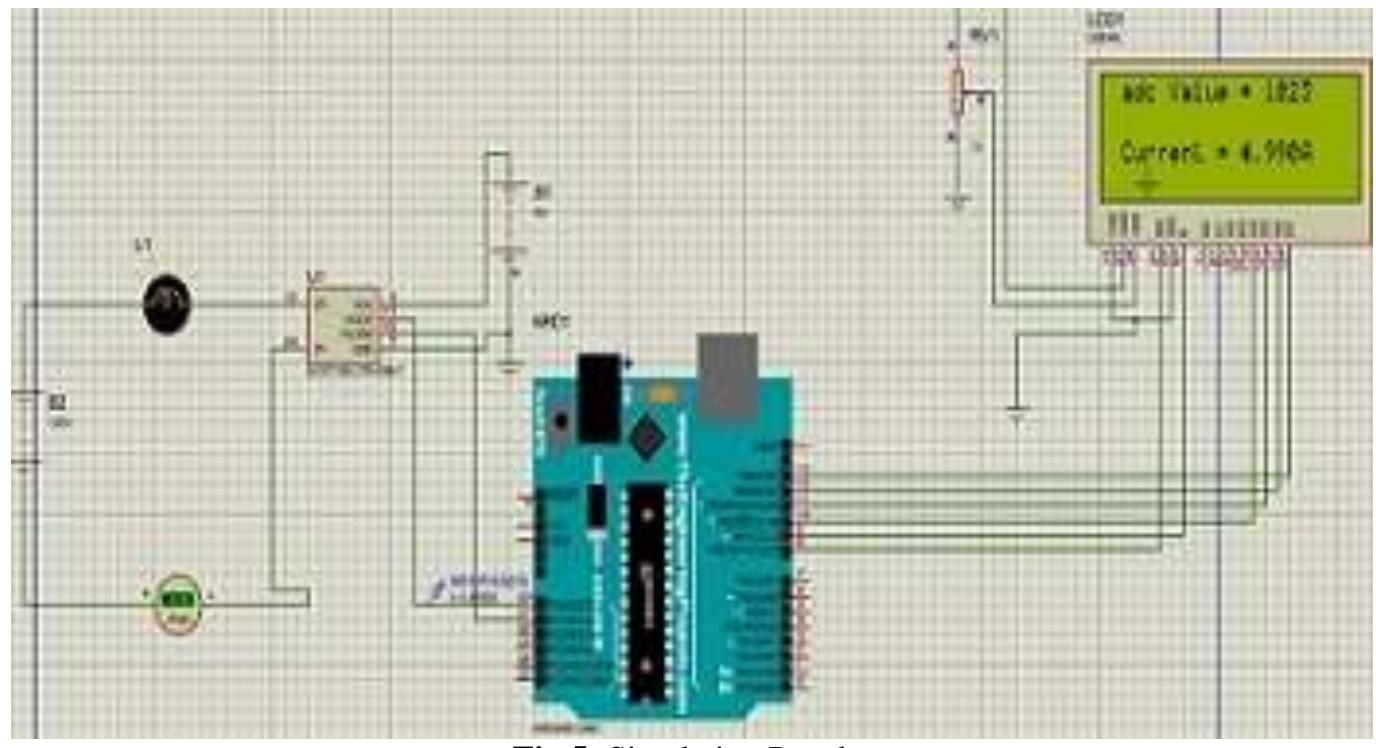

Fig 5: Simulation Results 


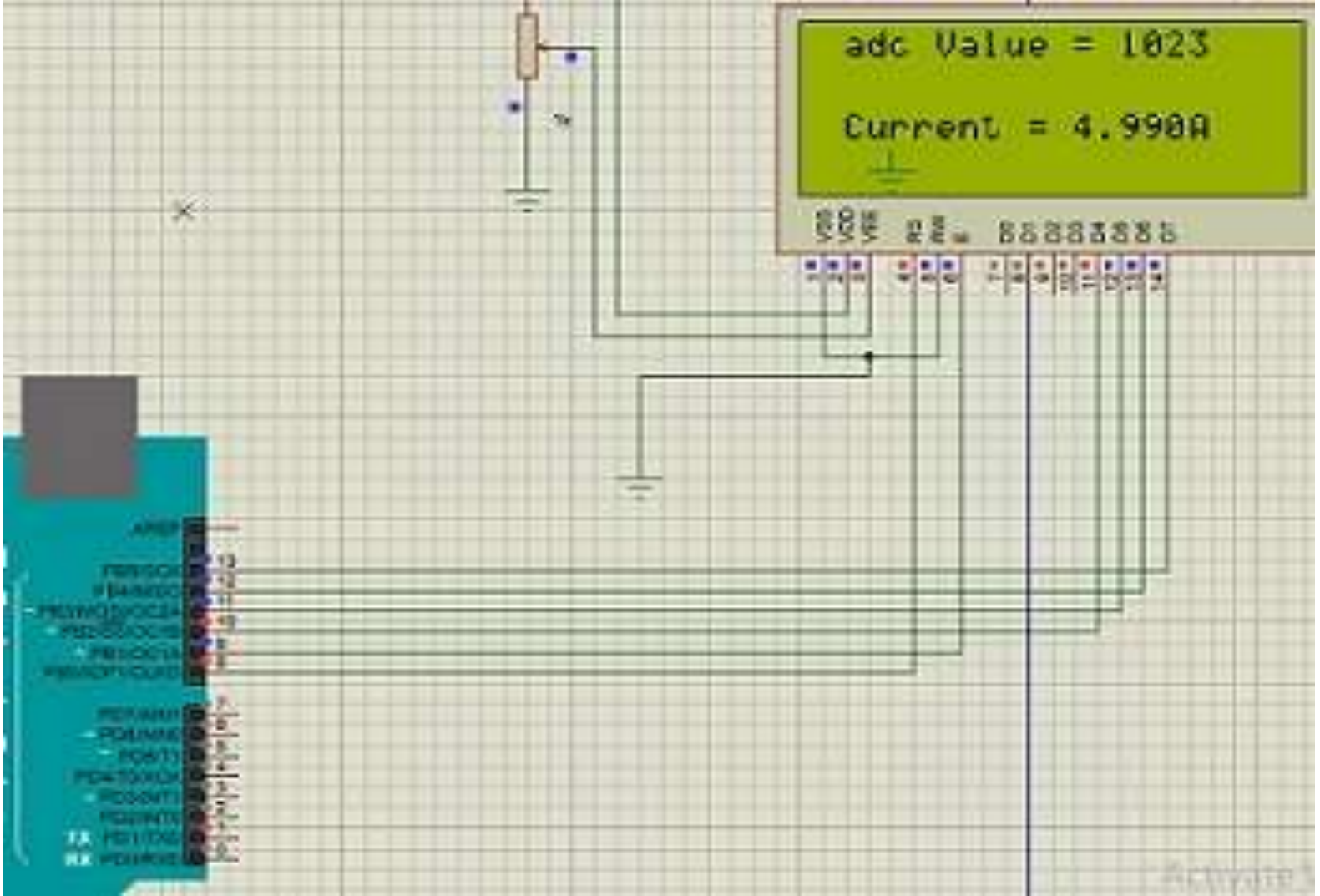

Fig 6: Current Sensing Output Display

\section{HARDWARE DESIGN}

Hardware design of the smart meter is implemented using arduino. Another important factor of the smart meter is that performance efficiency.

\subsection{Reason for Choosing Arduino Uno Controller}

The Advanced RISC Machines(ARM) are not binary compatible X86 processor which means it cannot run on windows faster because of this clock frequencies are limited [4]. And Digital Controllers has difficult and lengthy coding for the simple process itself where Arduino finds a suitable platform this application. Arduino is an open source physical processing hardware, which is based on a microcontroller board and an incorporated development environment for the board to be programmed. Arduino is simple and can run on any platform that includes Window Linux etc. unlike other microcontrollers, which run only in the Windows operating system.

The advantages of the Arduino are listed as follows.

1. Less expensive: Arduino boards are inexpensive compared to other microcontrollers that are available in the market. A preassembled Arduino board is available for as low.

2. Compatible: Arduino is compatible with all the operating systems including Linux, Macintosh, and Windows, whereas other microcontrollers are restricted to Windows.
3. Easy to program: The environment used to program Arduino and the ways to perform the coding are user friendly even for beginners.

4. Expandable programming and open source:

The programming language of an Arduino is an open source and can incorporate the Arduino code into the AVR-C code if needed.

\section{ARDUINO UNO}

Arduino Uno is a microcontroller board based on the Atmega 328. It has a ceramic Resonator that is $16 \mathrm{MHz}$, fourteen digital input/output pins (six of which can be used as PWM outputs), a reset button, a USB connection, a power jack and six analog inputs. It is an 8-bit microcontroller based on RISC architecture.

Microcontroller

- Atmega

328

Operating Voltage

Input Voltage (Recommended)

Input Voltage Limitation

Digital Input/output Pins

Analog Input Pins

Clock Speed

EEPROM

$-5 \mathrm{~V}$

- $7-12 \mathrm{~V}$

- $6-12 \mathrm{~V}$

$-14$

$-6$

- $16 \mathrm{MHz}$

- $1 \mathrm{~KB}$

The recommended voltage is between $7-12 \mathrm{~V}$ because if the voltage dips below $7 \mathrm{~V}$, the $5 \mathrm{~V}$ pin on the Arduino board will become unstable and if the voltage rises above $12 \mathrm{~V}$, the board may overheat and become damaged. 


\subsection{IoT Modem}

An IoT modem provides real-time monitoring with proper safety and security. The IoT webpage can be accessed using login to monitor the sensors values. IoT modem works in two modes: Auto and manual mode.

\section{THE FEATURES OF IoT MODEM INCLUDES}

1. One time configuration

2. Provides high transfer rate

3. Automatic mail and sms is sent to the user

4. Graph plot for all the sensor values with respect to time

6. Easy access of web server from anywhere anytime.

\section{Connection Diagram}

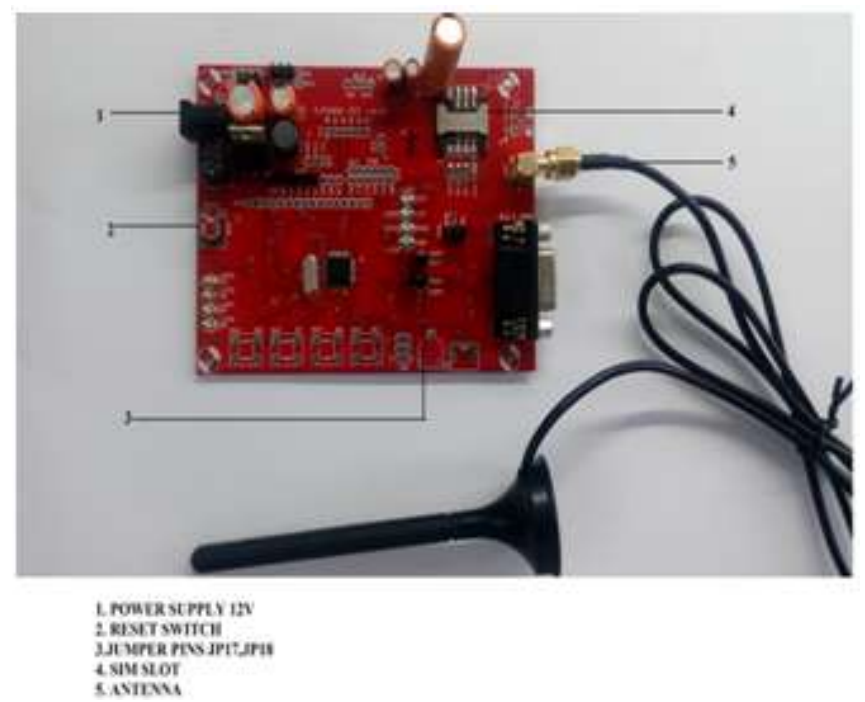

Fig 7: IoT MODEM V2 SIM 900

\subsection{LCD 1602 Display}

A liquid crystal display (commonly abbreviated LCD) is a thin, flat display device made up of any number of color or monochrome pixels arrayed in front of a light source or reflector. It is often utilized in battery-powered electronic devices because it uses very small amounts of electric power.

In this LM044L Liquid crystal display consists of $16 * 2$ Display size. The LCD display is used to display the processing results from the arduino and sensing unit.

\subsection{Current Sensor}

ZMCT103P current coil type sensor is used to measure load upto $5 \mathrm{~A}$.

The current transformer with the primary current input is upto $5 \mathrm{~A}$ and it can step down to a secondary of $2.5 \mathrm{~mA}$ which has a turns ratio of 1:1000 with the linearity of $0.1 \%$ and operating frequency is 50 to $60 \mathrm{~Hz}$ with the permissible error will be $-0.2 \%$ to $+0.2 \%$.

A0 is analog value which is going to read in the arduino
Current $=(($ Max current - Arduino measured analog reading $)$ *Ac Offset) /load in watts))

Voltage $=($ analog Read $(A 0) / 1024.0) * 5000$

Ac Offset is normal voltage output at Vout pin

Double RMS Current $=(($ Max Current -516$) * 0.707) / 100$

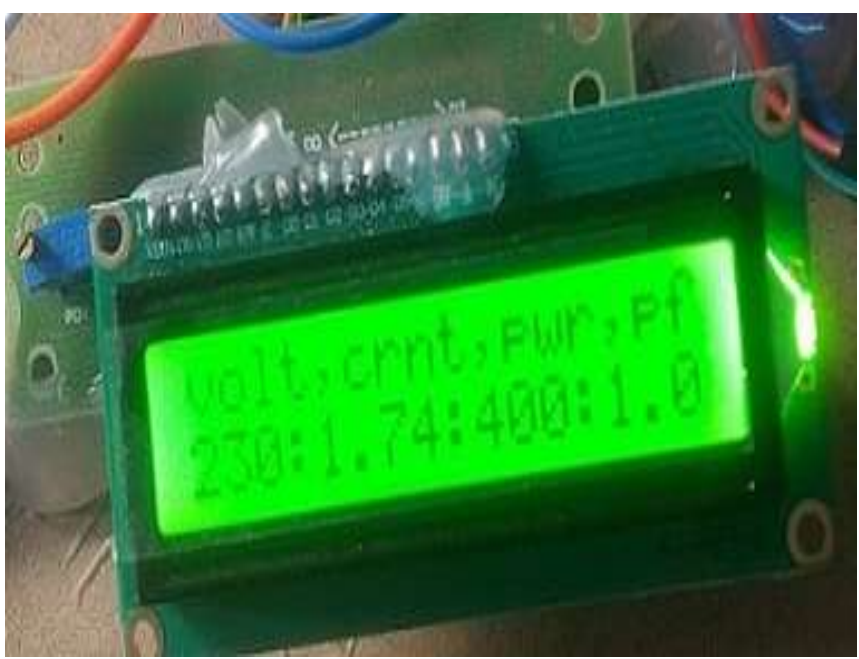

Fig 8: Power Consumption of the Lamp Load

The load of four $100 \mathrm{~W}$ is used and a supply voltage of single phase $230 \mathrm{~V}$ is given to it. The current which takes is around 3 A. Corresponding power, power factor are also calculated

Power is product of voltage (volt) and current (Amp)

$$
\mathrm{P}=\mathrm{V} \times \mathrm{I}
$$

Unit of power is $\mathrm{W}$ or $\mathrm{KW}$ In this way the current, voltage, power are calibrated.

\section{EXPERIMENTAL RESULTS AND ANALYSIS}

The hardware setup of the experiment which Consists of ammeter and multimeter to check the value from current sensor is shown in the Fig.9.

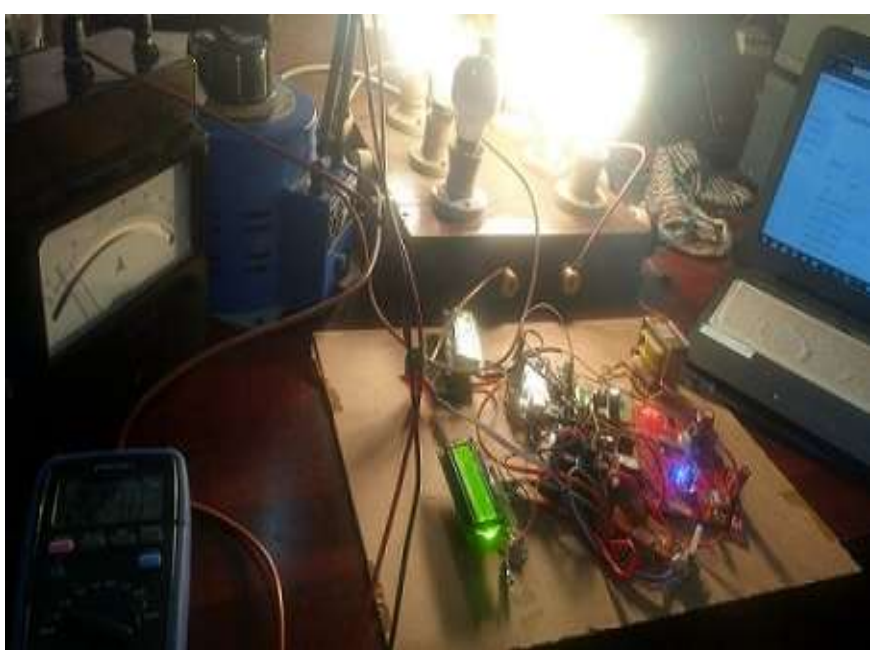

Fig 9: Hardware Setup 
The overall experimental setup circuit diagram can be shown in the fig.10. After current sensor, the arduino board and the IoT modem of our proposed model will be there.

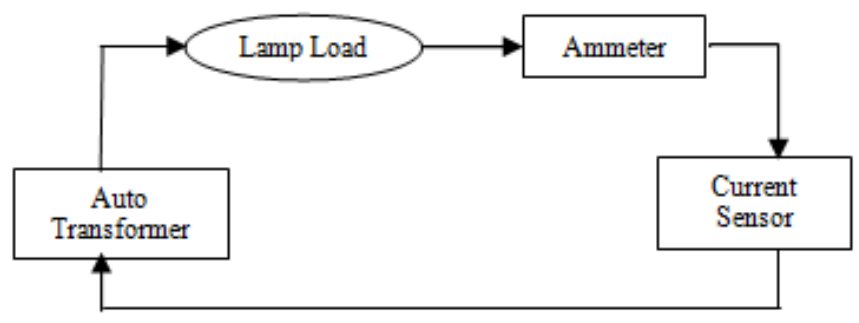

Fig 10: Overall Connection Diagram

At normal operating condition, power consumption of the respective load will be displayed in the respective smart meters. By-passing the data of main arduino through neighbouring arduino at the time of fault in shown in the Fig.11. As the main arduino to IoT modem connection gets disconnected.

The main arduino LCD display will be display as "connection barred". But at that time the data's are send through the neighbouring meter, the data's will not be displayed in it. It only display as "Routed"

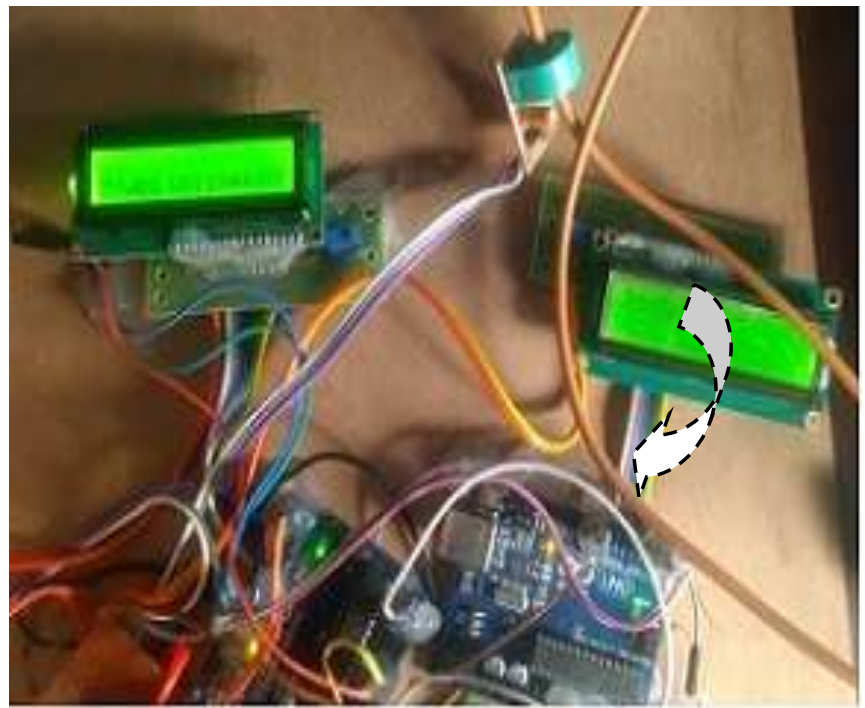

Fig 11: Bypassed the Load Data of Smart Meter

\section{CONCLUSION AND FUTURE WORK}

From the above it is evident that, one or more smart meters are interconnected with one another. If any one of the bus protocol of this meter gets damaged, the data's will be bypassed among them and send to the main server which also provides safety and security in the way that the data of default meter will not be visible to the adjacent smart meters. In this proposed ring system, the smart meters are connected to a single server of IoT modem thereby reduction in the huge amount of wiring to it and also minimize expenditure.
Further Modem to Control Center transmits data wirelessly. Usually power transmission is being carried out through wired connection and if the data of this power consumption is also transmitted through wired mode, will kills the process of smartness. So wireless transmission data is the need of the hour. This IoT modem based wireless transfer is to transmit data securely and smartly.

Hence from the above system it can be seen that the amount of power utilized by the consumer can be sensed and visualized to them. The future scope of the developed system is aimed at controlling the load along with its transferring of data and also displaying power consumption of consumer in an android application.

\section{REFERENCES}

[1] F. D. Garcia, F. P. Marafão, W. A. d. Souza and L. C. P. d. Silva, "Power Metering: History and Future Trends," 2017 Ninth Annual IEEE Green Technologies Conference (GreenTech), Denver, CO, 2017, pp. 26-33.

[2] E.J. Palacios-García et al., "Smart metering system for microgrids," IECON 2015 - 41st Annual Conference of the IEEE Industrial Electronics Society, Yokohama, 2015, pp. 003289-003294.

[3] Z. M. Fadlullah, et al., "An early warning system against malicious activities for smart grid communications", Network, IEEE, vol. 25, pp. 50-55, 2011.

[4] G. Aurilio, D. Gallo, C. Landi, M. Luiso, V. Cigolotti and G. Graditi, "Low cost combined voltage and current transducer for Smart Meters," 2014 IEEE International Instrumentation and Measurement Technology Conference (I2MTC) Proceedings, Montevideo, 2014, pp. 1459-1464.

[5] C. Landi, G. Del Prete, D. Gallo and M. Luiso, "The use of real-time instruments for smart power systems," 2012 IEEE International Energy Conference and Exhibition (ENERGYCON), Florence, 2012, pp. 884-889.

[6] R. Ramakrishnan and L. Gaur, "Smart electricity distribution in residential areas: Internet of Things (IoT) based advanced metering infrastructure and cloud analytics," 2016 International Conference on Internet of Things and Applications (IOTA), Pune, 2016, pp. 46-51.

[7] A.Molderink, V.Bakker, M.G.C.Bosman, J.L.Hurink, G.J.M.Smith "Management and Control of Domestic Smart Grid Technology", IEEE Tr. on. Is Date: Sept. 2010. Vol: 1 Iss.:2. pp: 109 - 119.

[8] K. Ramadan, E. Zakaria and D. M. Eltigani, "Prepaid energy meters network via power system communication," $2013 \quad$ INTERNATIONAL CONFERENCE ON COMPUTING, ELECTRICAL AND ELECTRONIC ENGINEERING (ICCEEE), Khartoum, 2013, pp. 668-673. 
[9] A.Molderink, V.Bakker, M.G.C.Bosman, J.L.Hurink, G.J.M.Smith "Management and Control of Domestic Smart Grid Technology", IEEE Tr. on. Is Date: Sept. 2010. Vol: 1 Iss.:2. pp: 109 - 119.

[10] E. Sacoto-Cabrera, J. Rodriguez-Bustamante, P. Gallegos-Segovia, G. Arevalo-Quishpi and G. LeónParedes, "Internet of Things: Informatic system for metering with communications MQTT over GPRS for smart meters," 2017 CHILEAN Conference on Electrical, Electronics Engineering, Information and Communication Technologies (CHILECON), Pucon, 2017, pp. 1-6.

[11] M. M. Haque, M. K. Hossain, M. M. Ali and M. R. I. Sheikh, "Microcontroller Based Single Phase Digital Prepaid Energy Meter for Improved Metering and Billing System," International Journal of Power Electronics and Drive System, vol. 1, no. 2, p. 139147, 2015.

[12] SIM900 AT Command List (Assessed on: 08.09.2014)

[Online].http://www.simcom.us/act_admin/supportfil e/SIM900_ATC_V1.00.pdf.

[13] F. Ciancetta, B. D'Apice, D. Gallo, C. Landi, "Plugn-Play Smart Sensor Based on Web Service", Ins. Tra.Inst. Meas. vol. 57, no. 10, pp. 2136- 2145, 200.

[14] W. Hlaing, S. Thepphaeng, V. Nontaboot, N. Tangsunantham, T. Sangsuwan and C. Pira, "Implementation of WiFi-based single phase smart meter for Internet of Things (IoT)," 2017 International Electrical Engineering Congress (iEECON), Pattaya, 2017, pp. 1-4.

[15] H. Y. Hsueh, C. Y. Lee, W. C. Liao and K. L. Kan, "Bi-directional and targeted AMI: Reference model based on internet-of-things," 2013 IEEE International Symposium on Consumer Electronics (ISCE), Hsinchu, 2013, pp. 165-166. 